\title{
Review of: "High efficiency multiplex biallelic heritable editing in Arabidopsis using an RNA virus"
}

\author{
Yongliang Zhang ${ }^{1}$ \\ 1 China Agricultural University
}

Potential competing interests: The author(s) declared that no potential competing interests exist.

In 2020, Ellison (Ellison et al., 2020) and coworkers developed a mobile RNA augmented TRV vector for sgRNA delivery and achieved highly efficient heritable genome editing in $N$. benthamiana. In this manuscript, authors extend that study by the development of new TRV vector for sgRNA delivery in Arabidopsis, multiplex and biallelic mutant progenies were obtained in one single generation. Except for the routinely used agroinfiltration method, authors proved that agro-pricking and agro-flooding methods also worked. Although Arabidopsis is easy to transform with the "floral dipping method", obtaining homozygous mutants still requires cumbersome screening. I think this work provides a good alternative to obtain Arabidopsis edits. To further enhance this study's thoroughness for publication in The Plant Physiology, I recommend the authors of this study address the following issues.

1. As homozygous or biallelic mutants count for the most desired edits, please determine the proportions of these mutants in the progenies in at least one target.

2. Have the authors conducted experiments to check whether TRV was transmitted to the next-generation or not, like RT-PCR analysis of randomly picked progenies?

3. I wonder whether the editing efficiency was affected by the relative position when two sgRNAs were arranged in tandem with a 23-base pair spacer? In figure $2 \mathrm{~A}$, if the sgRNA ${ }^{A t C H L I 2}$ was engineered in front of the sgRNA ${ }^{A t C H L I}$, what about the editing frequency compared to that shown in Figure 2B? 